\title{
Clinical and Etiological Profile of Mycotic Keratitis from a Tertiary Care Centre in South India
}

\author{
John Britto Augustin ${ }^{1}$, Sureshbaboo Variamkandi² \\ ${ }^{1}$ Family Health Centre, Aralam Farm, Kannur, Kerala, India, ${ }^{2}$ Department of Microbiology, \\ Government Medical College, Kozhikode, Kerala, India.
}

\section{ABSTRACT}

\section{BACKGROUND}

Corneal ulcer is the leading cause of ocular morbidity and monocular blindness worldwide. To effectively prevent blindness in patients with corneal ulcer, a proper understanding of the risk factors predisposing to ulceration, its clinical and microbiological characteristics are essential. Timely identification of aetiological agents causing corneal ulcers and their prompt treatment helps to save the vision. We wanted to detect aetiological agents of corneal ulcer with special references to fungal causes and characterize the fungal aetiological agents to species level.

\section{METHODS}

This is a cross sectional study, conducted in Government Medical college, Kozhikode, between January 2016 and June 2017. All patients who were clinically diagnosed as cases of infectious corneal ulcer in the Ophthalmology department, Government Medical College, Kozhikode were included in the study. Corneal scrapings collected from the infected eye were subjected to microbiological examination and culture. A total of 120 cases were analysed. Each patient was examined with the slit lamp bio microscope after staining with fluorescein. Scrapings from cornea at the site of corneal ulcer were collected by ophthalmologist after a detailed clinical history and examination of the affected eye. The laboratory procedures used in the diagnosis of infectious keratitis were based on direct visualization of organisms by subjecting corneal scrapings to Gram stain and $\mathrm{KOH}$ wet mount and inoculation of material on to blood agar and Sabouraud dextrose agar.

\section{RESULTS}

Among the 120 cases, a total of 49 cases were culture positive. Twenty-one [17.5 \%] were bacterial, twenty-two [18.34\%] were fungal and six [5.0\%] were poly microbial [bacteria and fungus]. Among the fungal aetiology, fusarium species was most common [32.14 \%], followed by aspergillus species - $25.0 \%$. Trauma was the major risk factor. Diabetes mellitus, exposure keratitis were the other comorbidities / risk factors.

\section{CONCLUSIONS}

This study shows majority of infected corneal ulcers are associated with risk factors and the aetiology are mainly fungi. Microscopy, culture, and clinical correlation helped in adequate management. Thus, prognosis and outcome of corneal ulcers rely on timely identification of their aetiology and prompt treatment.
Corresponding Author: Dr. Sureshbaboo Variamkandi, Additional Professor, Department of Microbiology, Government Medical College, Kozhikode, Kerala - 673008, India.

E-mail: sureshbaboovk@gmail.com

DOI: $10.14260 / \mathrm{jemds} / 2021 / 410$

How to Cite This Article:

Augustin JB, Variamkandi S. Clinical and etiological profile of mycotic keratitis from a tertiary care centre in South India. J Evolution Med Dent Sci 2021;10(27): 2001-2006, DOI:

10.14260/jemds/2021/410

Submission 05-03-2021,

Peer Review 08-05-2021,

Acceptance 15-05-2021,

Published 05-07-2021.

Copyright (c) 2021 John Britto Augustin et al. This is an open access article distributed under Creative Commons Attribution License [Attribution 4.0 International (CC BY 4.0)]

\section{KEY WORDS}

Corneal ulcer, Fungal Keratitis 


\section{BACKGROUND}

Corneal ulcer is a vision threatening disease and a leading cause of ocular morbidity and blindness worldwide.1,2 It is considered as an ophthalmic emergency which requires prompt treatment. The severity of corneal infection depends on the underlying condition of cornea and pathogenicity of the infecting organism. ${ }^{3}$ The incidence and spectrum of corneal ulcer depends on different factors, viz. geographical and location related factors, and the degree of development of a country. Corneal ulcer is the major cause of monocular blindness in developing countries. ${ }^{4}$ To effectively prevent blindness in patients with corneal ulcer, a proper understanding of risk factors predisposing to ulceration, and clinical and microbial characteristics of the disease is essential.

Infectious keratitis is a leading cause of corneal blindness in developing countries. It is a sight threatening disorder that affects both males and females across all age groups worldwide. Corneal ulceration results in $1.5-2$ million new cases of corneal blindness annually, posing a major public health problem according to the World Health Organization (WHO) reports. Fungi are the most common etiological agents which account for $30-40 \%$ whereas bacteria accounts for $13-48 \%$ of all cases of suppurative keratitis; this varies by geographical area.5,6 It is widely accepted that bacterial and fungal keratitis have higher incidence in developing world and viral keratitis is prevalent in developed countries [US]. Predominance of bacterial and fungal keratitis in developed countries have been reported now. ${ }^{1}$

Fungi are generally responsible for less than $10 \%$ of corneal infections in most clinical cases reported in the United States whereas in India, fungal keratitis accounts for more than $60 \%$ of the cases. ${ }^{7}$ When the integrity of ocular surface has been breached due to damage or trauma, it will be more vulnerable to be infected by fungi and other microbes resulting in fungal keratitis which is associated with traumatic corneal injury, especially from vegetative matter.

Fungal corneal ulcer is usually characterized by dry, raised slough, stromal infiltrates with feathery edges, satellite lesions and a thick [cheesy hypopyon] endothelial exudates.

The role of microbiologist in management of corneal ulcer is crucial as it has to be differentiated from other noninflammatory conditions of cornea resulting from immune mediated reactions.

Fungal corneal ulcer must be differentiated from bacterial corneal ulcer as the treatment varies. Diagnosis and treatment of corneal ulcer is challenging as the culture reports are sometimes inconclusive.

Microbial keratitis should be detected early and treated timely to save the vision. Ocular morbidity such as corneal scarring and subsequent visual loss can be significantly reduced by prompt institution of appropriate therapy guided by the knowledge of the causative agents. ${ }^{5}$ Severe corneal ulcers might progress to corneal perforation, with poor visual outcome. Therefore, identification of the pathogens and appropriate treatment is critical for a good clinical outcome. ${ }^{8}$

Treatment of microbial keratitis is difficult due to delayed presentation and difficulties in obtaining appropriate antifungal medications. Even with moderate corneal scarring, unilateral blindness is often the final result due to the lack of suitable donor material for a corneal graft. ${ }^{9}$

In this study we wanted to characterize the fungal etiological agents and the clinical outcomes.

\section{METHODS}

A cross sectional study was conducted at Ophthalmology and Microbiology departments of Government Medical College, Kozhikode between January 2016 and June 2017 after obtaining IRC approval. All patients clinically diagnosed with infectious corneal ulcers presenting to the Ophthalmology department of Government Medical College, Kozhikode were included in the study. Corneal ulcers of non-infectious aetiology were excluded from the study.

Each patient was examined with the slit lamp bio microscope by an ophthalmologist. After staining with fluorescein and with the variable slit on the bio microscope the details of the corneal ulcer including the location, size, shape, depth vascularization, and hypopyon were recorded. A sketch of each ulcer was also drawn on the casebook using standardized frontal and cross-sectional diagrams, and the presence or absence of a hypopyon was recorded and the size and height measured in millimetres. A standardized proforma was completed for each patient documenting sociodemographic information as well as clinical findings. Associated ocular conditions such as blepharitis, dacryocystitis, dry eyes, corneal anaesthesia or ocular leprosy were noted.10,11

Scrapings from cornea at the site of corneal ulcer were collected by ophthalmologist, after taking detailed clinical history, and examination of the affected eye. Eye was anesthetized with sterile $0.5 \%$ tetracaine or $4 \%$ lignocaine 2 - 3 drops. The surrounding skin was wiped with sterile gauze soaked in sterile saline and excess of discharge and debris were removed. A sterile self-retaining lid retractor was applied to minimize contamination with conjunctival flora. Using No. 15 Bard Parker blade, ulcers were scraped thoroughly and gently from base and edges of ulcer.12,13

The laboratory procedures used in the diagnosis of infectious keratitis were based on direct visualization of organisms in the material and inoculation of material on to blood agar and Sabouraud dextrose agar under appropriate conditions to allow multiplication of organisms. ${ }^{14}$

The scraped material was used to prepare a $10 \%$ potassium hydroxide wet mount and Gram's smear. KOH wet mount was examined for fungal hyphae [pseudohyphae, true hyphae, conidia].12,13,15,16 Gram stain was examined for pus cells, yeasts, and fungal hyphae. If hyphae were observed in Gram stain microscopy or KOH mount preparation, but failed to grow in culture, the aetiology was reported as fungal. ${ }^{17}$

Portion of scraped material was directly inoculated onto blood agar and Sabouraud dextrose agar media with antibiotics which included chloramphenicol \& gentamicin but without actidione [cycloheximide], 2 sets for isolation of fungi. ${ }^{14,15}$, All fungal cultures were incubated at $25^{\circ} \mathrm{C}$ and $37^{\circ}$ C separately for 4 weeks. ${ }^{17}$

Samples were streaked on culture plates as ' $\mathrm{C}$ ' or ' $\mathrm{S}$ ' shapes. All cultures were checked every day for first week 
and alternate days or twice weekly during next three weeks. The mycelial isolates were identified by their colony characteristics and microscopic appearance in Lactophenol Cotton Blue stained mount and finally by slide cultures (Fig. 1) The yeast isolates were identified by standard tests like germ tube, chlamydospores production on cornmeal agar, colonies on CHROMagar, urease test, sugar fermentation and assimilation. ${ }^{18}$ The criteria adopted for microbial evaluation was that the sample would be considered positive if any one of the following were met.

1. The growth of the same organism was demonstrated on two or more media on C - streak.

2. The same organism was grown from repeated scrapings.

3. It was consistent with clinical signs.

4. Smear results confirmed the finding from cultures.

\section{Statistical Analysis}

Data was entered in Epi infoTM Version 7.2, CDC, Atlanta, Georgia.

\section{RESULTS}

A total of 120 cases of infective corneal ulcer have been studied clinically and corneal scrapings of all the 120 cases were processed in Microbiology laboratory, Government Medical College, Kozhikode. Out of total 120 cases, 71 [59.17 $\%]$ were males and 49 [40.84 \%] were females. Age distribution of the cases is shown in Figure 1. $60-69$ yrs. age group was most commonly affected. Manual labourers (38.3 $\%$ ) accounted for majority of cases whereas $14.2 \%$ of cases were agricultural workers. Majority $(78.57 \%)$ had history of trauma of some kind. Table: 1 shows the objects that caused trauma.

\begin{tabular}{|ccc|}
\hline Objects of Trauma & $\begin{array}{c}\text { No. of Positive Fungal } \\
\text { Cases }\end{array}$ & Percentage \\
\hline Unknown foreign body exposure & 10 & 35.71 \\
Vegetative matter, wooden twigs, & 9 & 32.14 \\
sticks & 2 & 7.14 \\
Concrete particles and cement & 1 & 3.57 \\
Metal pieces & 6 & 21.43 \\
No trauma & $\mathbf{2 8}$ & $\mathbf{1 0 0}$ \\
\hline Total & Table.1 Objects of Trauma \\
\hline
\end{tabular}

Diabetes mellitus and hypertension were the most common comorbidities associated with those cases where there was no history of trauma. No history of trauma in $6 / 28$ [21.43\%] cases. The most common symptoms were pain [86.7 \%] and redness [75\%] followed by watering [50 \%].
Prior treatment with steroid lead to fungal corneal ulcer in one case. 26 out of 28 [92.9\%] fungal corneal ulcers had size less than $6 \mathrm{~mm}$. All fungal ulcers involved stroma. All fungal ulcers had irregular margins. Ulcers were mostly central than peripheral. There were 35 suspected cases of fungal keratitis of which 22 cases fungi were isolated in pure. In 6 cases fungi grew along with bacteria. In 7 cases fungal elements were seen only by microscopy and failed to grow by culture. Of the 22 cases, fungi were the sole etiological agents for keratitis, fungi could be demonstrated both by microscopy and culture. In 17 cases fungi were demonstrated only by culture. In six cases fungal growth was obtained along with bacteria. In two of these cases, presence of fungi could be established both by microscopy and culture. In the rest, fungi could be demonstrated only by culture.

\section{Microbiological Profile of Corneal Ulcer}

Table 2 shows the microbiological profile of etiological agents of corneal ulcers in the current study.

\begin{tabular}{|ccc|}
\hline Isolates & No. of Cases & Percentage \\
Bacteria & 21 & $17.5 \%$ \\
Fungus & 22 & $18.34 \%$ \\
Parasite & 0 & $0 \%$ \\
Mixed isolates [bacterial + & 6 & $5 \%$ \\
fungal] & 71 & $59.17 \%$ \\
Organism not isolated & 120 & $100.0 \%$ \\
\hline Overall total & Table 2. Microbiological Profile of Corneal Ulcer \\
\hline
\end{tabular}

\section{Total Fungal Profile}

Most common fungal isolate was fusarium spp [32.14\%]. Table 4 shows the profile of microbes obtained from corneal ulcers where fungi and bacteria were isolated together.

\begin{tabular}{|c|c|c|c|c|c|c|}
\hline Fungal Isolates & $\begin{array}{c}\text { Pure } \\
\text { Isolates }\end{array}$ & $\%$ & $\begin{array}{l}\text { Mixed } \\
\text { Isolate }\end{array}$ & $\%$ & $\begin{array}{l}\text { Total No. } \\
\text { of Cases }\end{array}$ & $\%$ \\
\hline Fusarium solani & 3 & \multirow{3}{*}{5.84} & 2 & \multirow{3}{*}{1.7} & 5 & \multirow{3}{*}{7.5} \\
\hline Fusarium oxysporum & 4 & & 0 & & 4 & \\
\hline Total & 7 & & 2 & & 9 & \\
\hline Aspergillus flavus & 3 & \multirow{4}{*}{5} & 0 & \multirow{4}{*}{0.8} & 3 & \multirow{4}{*}{5.9} \\
\hline Aspergillus terreus & 2 & & 0 & & 2 & \\
\hline Aspergillus fumigates & 1 & & 1 & & 2 & \\
\hline Total & 6 & & 1 & & 7 & \\
\hline Aureobasidium pullulans & 4 & 3.34 & 0 & 0 & 4 & 3.3 \\
\hline $\begin{array}{l}\text { scedosporium } \\
\text { apiospermum }\end{array}$ & 1 & 0.83 & 1 & 0.8 & 2 & 1.7 \\
\hline acremonium spp & 1 & 0.83 & 1 & 0.8 & 2 & 1.7 \\
\hline Candida albicans & 1 & 0.83 & 0 & 0 & 1 & 0.8 \\
\hline cylindrocarpon spp & 0 & 0 & 1 & 0.8 & 1 & 0.8 \\
\hline Fonsecaea pedrosoi & 1 & 0.83 & 0 & 0 & 1 & 0.8 \\
\hline mucor spp & 1 & 0.83 & 0 & 0 & 1 & 0.8 \\
\hline Total & 22 & 18.33 & 6 & 4.9 & 28 & 23.3 \\
\hline
\end{tabular}

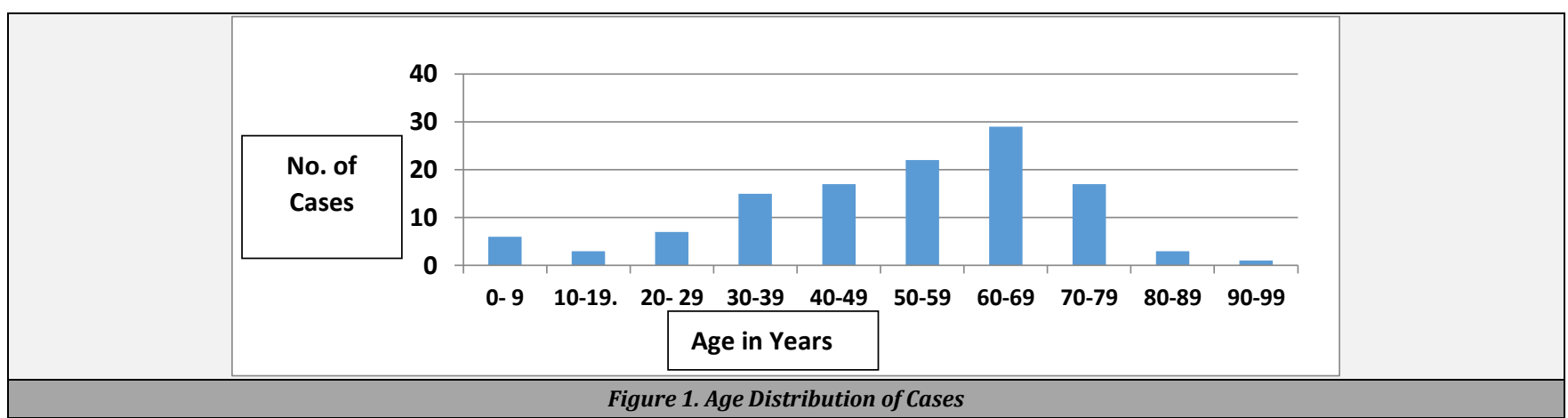




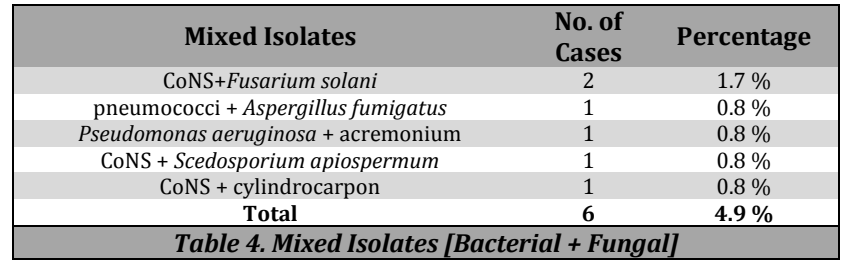

Aetiology Vs Clinical outcome

\begin{tabular}{|cccc|}
\hline Aetiology & Improved & $\begin{array}{c}\text { Recurrence / } \\
\text { Complication }\end{array}$ & Total \\
Fungal & 21 & 1 & 22 \\
$\begin{array}{c}\text { Mixed } \\
\text { [Bacterial +fungal] }\end{array}$ & 5 & 1 & 6 \\
Not isolated / sterile & 0 & 0 & 92 \\
Total & $\mathbf{2 6}$ & $\mathbf{2}$ & $\mathbf{1 2 0}$ \\
\hline \multicolumn{4}{|c}{ Table 5. Aetiology and Clinical Outcome } \\
\hline
\end{tabular}

Of the 28 cases where fungal aetiology was proved, 26 improved clinically and 2 had recurrence / complications. None of the cases healed completely. All seven suspected cases, where fungal elements were seen only by microscopy and not culture, healed well.

\section{DISCUSSION}

A total of 120 clinically diagnosed cases of corneal ulcers were studied. Demographic details, aetiology, risk factors, fungal profile and clinical outcomes were recorded. In this study, cases belonged to different age groups from 0 - 99 years. Median age was 55 and higher incidence was noted in 60 - 69 years. Males in age group 50 - 59 years, were most commonly affected, which might be due to more outdoor activity. The cases were most common amongst manual labourers accounting to increased ocular trauma in such cases.

A definite relationship between corneal injury and mycotic keratitis was observed. About $78.57 \%$ of all fungal corneal ulcers [22 out of 28 positive fungal corneal ulcer cases] had history of corneal injury. Meena and Sharma in their study of fungal corneal ulcer observed that majority of patients had trauma to the eye as most common predisposing factor. ${ }^{19}$ Gopinathan et al. found that ocular trauma predisposed to infection in $736(54.4 \%)$ of 1,354 eyes $^{20}$ in this study. Unknown foreign body exposure [35.71\%], trauma due to vegetative matter and wooden twigs and sticks [32.14\%], followed by concrete particles and cement [7.14 $\%$ ] and finally metal pieces [3.57\%] were the common objects of trauma. Diabetes mellitus was an important risk factor in injured and non-injured cornea. 6 out of 28 [21.43 $\%$ ] cases had no history of trauma.

Peponis et al. analysed that the cases in their study suggested that in patients with fungal keratitis who previously received topical corticosteroids, the abrupt cessation of these agents was likely to lead to an acute rebound inflammatory reaction and even perforation. ${ }^{21}$ Among our study population two patients received steroid therapy prior to the onset of ulcer and one of them developed fungal ulcer.

Pain and redness were the common symptoms in this study followed by watering, photophobia, foreign body sensation, defective vision. Fungal ulcers [pure and mixed isolate] involved corneal stroma in all cases. All corneal ulcers by fungi and mixed isolates had irregular margins. Clinical examination of corneal ulcer, especially margins give a clue to diagnosis by predicting its aetiology. Ravinder et al. observed that, fungal culture was positive in majority of cases with satellite lesions, thick, large hypopyon, irregular infiltrate margins, and endothelial infiltrate. ${ }^{22}$ In this study, central corneal ulcers were more common, than inferotemporal.

Of the 22 pure fungal cases, five were positive by microscopy and culture and 17 were microscopy negative but culture positive. In case of mixed isolates, out of six, two were microscopy and culture positive, four were microscopy negative but culture positive. In 7 cases, fungal elements could be demonstrated only by microscopy. This might be due to prior antifungal therapy and scanty scraping material. Gram stain positivity was $5.7 \%$ of fungal elements in 35 suspected fungal cases. $\mathrm{KOH}$ positivity for fungus was $34.3 \%$ [12 out of 35 cases]. Out of 120 cases, 28 cases [23.34\%] had definite fungal aetiology. The seven cases where fungal elements were seen only by microscopy were treated as mycotic keratitis. Mixed growth of fungi and bacteria were obtained from 6 cases. The mixed isolates were Streptococcus pneumonia + Aspergillus fumigatus - 1 out of 6 [16.67\%], Pseudomonas aeruginosa + acremonium - 1 [16.67\%], CoNS + Fusarium solani - 2 [33.34\%], CoNS + Scedosporium apiospermum - 1 [16.67\%], CoNS + cylindrocarpon - 1 [16.67 $\%$ ]. Mixed ulcers had irregular margins and all involved stroma, one among them was perforated.

Fusarium spp accounted for $32.14 \%$ of keratitis in the present study. Six out of nine cases, had history of trauma and one had diabetes mellitus. In 2 cases fusarium spp grew along with bacteria [CoNS], and one among them had prior steroid treatment history. All 9 cases had ulcer of size $<6 \mathrm{~mm}$, except one. Margins of the ulcers were irregular and involved stroma. $55.56 \%$ of cases had hypopyon. Further morphology was confirmed by slide culture and LPCB mount. 5 were Fusarium solani and 4 were Fusarium oxysporum. Ulcers were managed by repeated scrapings [debridement], topical natamycin and oral fluconazole. Antibiotics were given for mixed isolate with bacteria and to prevent secondary infection. Hassan et al. observed that, fusarium species were the most common cause of fungal infection in Southern US ( $45 \%-76 \%$ of fungal keratitis). ${ }^{23}$ Leck et al. observed, that fusarium spp and aspergillus spp were isolated from $61 \%$ of all fungal infections and comprised $83 \%$ of identified fungal isolates, although fusarium species were the most prevalent fungal pathogens reported in Ghana. Fusarium spp have also been found to be the principal fungal pathogen in Florida, Paraguay, Nigeria, Tanzania, Hong Kong, and Singapore. ${ }^{24} \mathrm{Wu}$ et al. noted that, currently, natamycin is considered to be the most effective topical agent against fusarium and aspergillus and natamycin is more effective than voriconazole in treatment of fungal keratitis, especially Fusarium keratitis. ${ }^{25}$

$25 \%$ of all keratitis was caused by aspergillus spp. Six out of seven aspergillus keratitis cases had history of trauma [mainly due to vegetative objects, soil], 2 had diabetes mellitus as additional risk factor. One of them was along with bacterial growth [pneumococci]. Ulcers were $<6 \mathrm{~mm}$, with irregular margins, central, paracentral or peripheral ulcers, involving stroma. Of the seven aspergillus spp isolated, 3 were Aspergillus flavus, 2 Aspergillus terreus, 2 Aspergillus fumigatus. All ulcers were treated with debridement, natamycin and antibiotics. Ulcers improved except one, which had recurrence and complications, with a bad prognosis. 
Khairallah et al. observed that the high prevalence of aspergillus spp. in their study, may be due to the fact that spores of aspergillus can survive the hot and dry weather of Saudi Arabia, and four of the 27 patients developed fungal endophthalmitis. ${ }^{26}$

All 4 Aureobasidium pullulans keratitis cases had history of trauma. Ulcers were $<6 \mathrm{~mm}$, irregular, stroma involved, central or peripheral with one perforated ulcer. All ulcers improved when they were treated by repeated scraping, natamycin and fluconazole. Aureobasidium is considered to be contaminant, but is potential to cause corneal ulcers. Panda et al. observed that, of 200 fungal organisms isolated from the cultures, 25 were identified as A. Pullulans. Of 25 eyes, 22 responded well to antifungal therapy and 2 required therapeutic penetrating keratoplasty and one patient progressed to phthisis bulbi. ${ }^{27}$

Out of the 2 Scedosporium apiospermum keratitis cases, one had history of trauma [isolated along with CoNS]. Ulcers were $<6 \mathrm{~mm}$ with irregular margins, paracentral ulcers involving stroma. Ulcers improved on repeated scraping, natamycin, fluconazole and antibiotics. Malla reddy et al. observed that, 3 out of 36 fungal isolates were Pseudallescheria boydii ( $8.33 \%$ ) in their study. ${ }^{28}$ Nulens et al. noticed that voriconazole, together with penetrating keratoplasty, was effective for the treatment of $S$. apiospermum keratitis. ${ }^{29}$

Both the acremonium [cephalosporium] keratitis cases had history of trauma, one was isolated along with Pseudomonas aeruginosa. Ulcers were, $<6 \mathrm{~mm}$ with irregular margins, central or peripheral and stroma involved. Empirical antibiotics were started initially, and repeated scraping was done, natamycin was started after the microscopy reports. Ulcers improved. Kim et al. noted that management of fungal corneal ulcers can be difficult, particularly as there are no standard therapies for infections caused by acremonium. Nowadays, topical, and oral voriconazole have been reported to be effective for the treatment of fungal keratitis. ${ }^{30}$

One Candida albicans had been isolated from 72-year-old, who had no history of trauma, but exposure keratitis and diabetes mellitus as risk factors. Ulcer was $<6 \mathrm{~mm}$ with irregular margins, central ulcer and involved stroma. Ulcer was initially treated with antibiotics, later based on culture reports, natamycin and fluconazole were added, and ulcer improved. Hassan et al. observed that, candida and aspergillus species were most common in Northern US and Candida albicans keratitis is a challenge for ophthalmologists due to its tendency to mimic other conditions. ${ }^{23}$ Candida species are the most common fungal organisms causing keratitis in HIV - positive patients. ${ }^{23}$

$3.57 \%$ of total fungal keratitis isolated are Fonsecaea pedrosoi, which had no history of trauma. Ulcer was $<6 \mathrm{~mm}$, with irregular margins, central ulcer and stroma involved. Since dematiaceous fungi takes more time to grow on culture media, ulcer was managed by antibiotics initially. Based on culture reports, fluconazole and natamycin were added and debridement was done. Ulcer improved. Chaidaroon et al. noticed that, F. pedrosoi is a rare cause of corneal infection. Although the characteristic visible pigmented infiltrate can be an important diagnostic clue, this classical finding may not be apparent in all cases. Therefore, it is important to establish an accurate diagnosis through microscopic evaluation and culture. In this study, the treatment with topical antifungal agents (ketoconazole and natamycin) and oral itraconazole resulted in the slow eradication of the infections. ${ }^{31}$

One cylindrocarpon species was isolated along with CoNS, which had history of trauma with white cement. Ulcer was > $6 \mathrm{~mm}$, with irregular margins, central ulcer and involved stroma and endothelium, to form corneal abscess. Moxifloxacin topical was started empirically, and natamycin and oral fluconazole on smear and culture results. Ulcer worsened and voriconazole was added as intracameral, intralesional and as a topical. Ulcer improved with no further deterioration. Irek et al. observed that, keratomycosis caused by $C$. lichenicola is rare in humans and challenging to manage. Although voriconazole has been used with success in some reports in managing $C$. lichenicola, its accessibility is limited. ${ }^{32}$

Mucor was isolated from a single case in the present study and had history of trauma with no diabetes. Ulcer was < $6 \mathrm{~mm}$, with irregular margins, central ulcer with stroma involved. Topical itraconazole was added based on culture reports along with antibiotics. Ulcer improved. Sanjeev et al. observed that, one $(03.86 \%)$ out of 26 isolates were mucor species, from a total of 127 cases suspected to have fungal keratitis. 33

\section{CONCLUSIONS}

To conclude, fungal ulcer shows gradual presentation and can be aggressive with delay in prompt management. Fungal corneal ulcer is difficult to treat and has become a challenge to microbiologists for identification and ophthalmologists to treat. Therefore, early identification and prompt timely treatment of corneal ulcer gives good clinical outcome, thereby saving the vision.

Data sharing statement provided by the authors is available with the full text of this article at jemds.com.

Financial or other competing interests: None.

Disclosure forms provided by the authors are available with the full text of this article at jemds.com.

\section{REFERENCES}

[1] Ibrahim YW, Boase DL, Cree IA. Incidence of infectious corneal ulcers: portsmouth study, UK. J Clin Exp Ophthalmol 2012: p. 1.

[2] Sharma K, Mehta S, Singh VA, et al. A profile of corneal ulcers-2 years study from rural hospital, Haryana. Journal of Dental and Medical Sciences 2014;13(11):947.

[3] Bourcier T, Thomas F, Borderie V, et al. Bacterial keratitis: predisposing factors, clinical and microbiological review of 300 cases. Br J Ophthalmol 2003;87(7):834-8.

[4] Bharathi MJ, Ramakrishnan R, Vasu S, et al. Aetiological diagnosis of microbial keratitis in South India-a study of 1618 cases. Indian J Med Microbiol 2002;20(1):19-24.

[5] Ranjini CY, Waddepally VV. Microbial profile of corneal ulcers in a tertiary care hospital in South India. J Ophthalmic Vis Res 2016;11(4):363-7.

[6] Suwal S, Bhandari D, Thapa P, et al. Microbiological profile of corneal ulcer cases diagnosed in a tertiary care 
ophthalmological institute in Nepal. BMC Ophthalmol 2016;16(1):1-6.

[7] Saratha D. Predisposing factors and microbiological profile of corneal ulcer. Sch J Appl Med Sci 2017;5(5B):1794-800.

[8] Kampitak K, Suntisetsin H, Sirikul T. Clinical and microbiological characteristics of corneal ulcers in a Thai referral center. Asian Biomed 2014;8(2):275-82.

[9] Prakash S, Kamisetty R. Prospective evaluation of the clinical profile, treatment outcomes and the demand for corneal transplantation in microbial keratitis: a single center analysis. 2015. http://imsear.li.mahidol.ac.th/handle/123456789/1768 30.

[10] Srinivasan M, Gonzales CA, George C, et al. Epidemiology and aetiological diagnosis of corneal ulceration in Madurai, South India. Br J Ophthalmol 1997;81(11):96571.

[11] Geethakumari PV, Remya R, Girijadevi PS, et al. Bacterial keratitis and fungal keratitis in South Kerala: a comparative study. Kerla J Ophthalmol 2011;23(1):43-6.

[12] Pakzad-Vaezi K, Levasseur SD, Schendel S, et al. The corneal ulcer one-touch study: a simplified microbiological specimen collection method. Am J Ophthalmol 2015;159(1):37-43.e1.

[13] Kunwar M, Adhikari RK, Karki DB. Microbial flora of corneal ulcer and their drugs sensitivity. Med J Shree Birendra Hosp 2013;12(1):14-7.

[14] Garg P, Rao GN. Corneal ulcer: diagnosis and management. Community Eye Health 1999;12(30):21-3.

[15] Gandhi S, Shakya D, Ranjan K, et al. Corneal ulcer: a prospective clinical and microbiological study. Int J Med Sci Public Health 2014;3(11):1334-7.

[16] Mascarenhas J, Lalitha P, Prajna NV, et al. Acanthamoeba, fungal and bacterial keratitis: a comparison of risk factors and clinical features. Am J Ophthalmol 2014;157(1):56-62.

[17] Chander J. Text book of Medical Mycology. $4^{\text {th }}$ edn. New Delhi: Jaypee Brothers Publishers 2018.

[18] Riaz Q, Fawwad U, Bhatti N, et al. Epidemiology of microbial keratitis in a tertiary care center in Karachi. Pak J Ophthalmol 2013;29(2):94-9.

[19] Meena V. A study of fungal corneal ulcer. Int J Med Res Rev 2016;4(3):324-9.

[20] Gopinathan U, Garg P, Fernandes M, et al. The epidemiological features and laboratory results of fungal keratitis: a 10-year review at a referral eye care center in South India. Cornea 2002;21(6):555-9.

[21] Peponis V, Herz JB, Kaufman HE. The role of corticosteroids in fungal keratitis: a different view. $\mathrm{Br} \mathrm{J}$ Ophthalmol 2004;88(9):1227.

[22] Ravinder K, Madhav MV, Archana J, et al. Clinical evaluation of corneal ulcer among patients attending teaching hospital. International Journal of Contemporary Medical Research 2016;3(4):949-52.

[23] Hassan HMJ, Papanikolaou T, Mariatos G, et al. Candida albicans keratitis in an immunocompromised patient. Clin Ophthalmol 2010;4:1211-5.

[24] Leck AK, Thomas PA, Hagan M, et al. Aetiology of suppurative corneal ulcers in Ghana and South India and epidemiology of fungal keratitis. Br J Ophthalmol 2002;86(11):1211-5.

[25] Wu J, Zhang WS, Zhao J, et al. Review of clinical and basic approaches of fungal keratitis. Int $\mathrm{J}$ Ophthalmol 2016;9(11):1676-83.

[26] Khairallah SH, Byrne KA, Tabbara KF. Fungal keratitis in Saudi Arabia. Doc Ophthalmol 1992;79(3):269-76.

[27] Panda A, Das H, Deb M, et al. Aureobasidium pullulans keratitis. Clin Exp Ophthalmol 2006;34(3):260-4.

[28] Mallareddy US, Katay P, Ravi S. A Study of infective keratitis and its response to treatment. Journal of Dental and Medical Sciences 2016;15(5):72-6.

[29] Nulens E, Eggink C, Rijs AJMM, et al. Keratitis caused by scedosporium apiospermum successfully treated with a cornea transplant and voriconazole. J Clin Microbiol 2003;41(5):2261-4.

[30] Kim SJ, Cho YW, Seo SW, et al. Clinical experiences in fungal keratitis caused by Acremonium. Clin Ophthalmol 2014;8:283-7.

[31] Chaidaroon W, Tananuvat N, Chavengsaksongkram P, et al. Corneal chromoblastomycosis caused by fonsecaea pedrosoi. Case Rep Ophthalmol 2015;6(1):82-7.

[32] Irek EO, Obadare TO, Udonwa PA, et al. Cylindrocarpon lichenicola keratomycosis in Nigeria: the challenge of limited access to effective antimicrobials. Afr J Lab Med 2017;6(1):612.

[33] Sanjeev H, Karnaker VK, Vijay P, et al. Fungal profile of infectious keratitis in a tertiary care hospital-our experience. Nitte University Journal of Health Science (NUJHS) 2012;2(2):10-4. 